\title{
Volume correction in the aging hand: role of dermal fillers
}

\author{
Alexander Z Rivkin \\ David Geffen/UCLA School of \\ Medicine Los Angeles, CA, USA
}

This article was published in the following Dove Press journal:

Clinical, Cosmetic and Investigational Dermatology

30 August 2016

Number of times this article has been viewed
Correspondence: Alexander Z Rivkin David Geffen/UCLA School of Medicine, I I 645 Wilshire Blvd, Ste 800,

Los Angeles, CA 90025, USA

Tel + I 3104435273

Email arivkin9@gmail.com
Abstract: The hands, just like the face, are highly visible parts of the body. They age at a similar rate and demonstrate comparable changes with time, sun damage, and smoking. Loss of volume in the hands exposes underlying tendons, veins, and bony prominences. Rejuvenation of the hands with dermal fillers is a procedure with high patient satisfaction and relatively low risk for complications. This study will review relevant anatomy, injection technique, clinical safety, and efficacy of dermal filler volumization of the aging hand.

Keywords: dermal fillers, hands, volumization, hyaluronic acid, calcium hydroxylapatite

\section{Introduction}

Rejuvenation of aging hands has become increasingly popular in cosmetic medicine over the last 8 years. Along with the face and neck, the hands are the most visible parts of the body and undergo signs of aging similarly. Just like in the face, volume loss in the hands is a common indicator of age.

Both intrinsic and extrinsic factors influence aging of the hands. The epidermis of the skin thins; lentigines, dyschromia, and textural roughness appear; and seborrheic and/or actinic keratosis may develop. The quantity and quality of collagen and elastin decline in the dermis, resulting in skin laxity and thinning. Deep to the skin, atrophy of the subcutaneous fat causes underlying tendons and bony prominences to become visible. The speed and extent of the changes varies with the individual patient's genetics. The progression can also be influenced by factors such as smoking and alcohol abuse, chronic sun exposure, overwork, chemical toxin exposure, and rheumatologic disease. ${ }^{1}$ Treatments to rejuvenate the hands, published in the literature, include skin excision, lasers and peels for skin resurfacing, sclerotherapy to reduce noticeable veins, and the use of autologous fat and/or fillers for volumization. ${ }^{2}$

\section{Pharmacology}

The use of autologous fat for hand rejuvenation was first described by Fournier in 1988 , using a single large bolus that was then massaged over the dorsal surface. ${ }^{3}$ Years later Coleman modified his facial fat grafting technique and emphasized blunt tunneling using smaller boluses which he believed increased smoothness and fat retention. ${ }^{4}$ With the commercial availability of hyaluronic acid (HA) and calcium hydroxylapatite (CaHA) fillers, many physicians began to use fillers instead of fat due to ease of use and consistency of results. These products were being used off-label, but results were found to be excellent, and hand augmentation with fillers became popular throughout 
the world. In June 2015, Merz Aesthetics reported that its CaHA product (Radiesse ${ }^{\circledR}$; Merz Aesthetics, Franksville, WI, USA) was the first FDA-approved injectable filler for hand augmentation to correct volume loss in the dorsum of the hands.

CaHA as a dermal filler is an injectable containing 30\% CaHA microparticles in 70\% aqueous carboxymethylcellulose gel carrier. ${ }^{5}$ The particles are relatively large, ranging from 25 to $45 \mu \mathrm{m}$ in size, which limits macrophage phagocytosis and provides durability. After injection, the carrier gel is broken down by the body within 2-3 months but the CaHA remains, stimulating fibroblast ingrowth and formation of collagen around the microspheres. ${ }^{6,7} \mathrm{CaHA}$ is chemically identical to the calcium component of bones and teeth, giving the product a high degree of biocompatibility. ${ }^{8}$ The safety profile of CaHA is well established, since the material has been in wide use for several decades. ${ }^{9}$ Allergy testing is not required, and clinical data demonstrate that the material does not stimulate calcification or a foreign body reaction. ${ }^{10}$

The first cosmetic indication that $\mathrm{CaHA}$ received from the FDA was for the correction of moderate-to-severe lines and wrinkles in 2006. Because of the firmness of the product and the duration of effect ( 6 months per the FDA indication, but it has been reported to last up to 2 years by some authors ${ }^{11}$ ), CaHA has been described as an injectable implant rather than a wrinkle filler. ${ }^{5}$

HA as a dermal filler first became available in December 2003. HA is a glucosaminoglycan biopolymer composed of strands of repeating chains of d-glucuronic acid and $N$-acetyld-glucosamine. ${ }^{12}$ It is a widely encountered component of the extracellular matrix in humans as well as many other species. HA is present in the skin, synovial fluid, muscle, and vitreous body of the eye, among other places in the human body. ${ }^{13}$ As a lubricant and an important structural component of tissues, HA is extremely hydrophilic and can bind many times its own weight in water. ${ }^{12} \mathrm{HA}$ is also highly biocompatible with a low incidence of antigenic adverse events (AEs). ${ }^{12}$

Native HA has low viscosity and a very short half-life (one-third of endogenous HA is recycled daily). ${ }^{13}$ In order to increase the duration, HA filler manufacturers incorporate cross-linking agents to make the products dense and durable enough to be effective filling agents. The firmness and longevity of commercially available HA products vary according to HA concentration, molecular weight of the $\mathrm{HA}$, and extent of cross-linking, with duration ranging from 6 months to 2 years. ${ }^{12}$ Early HA products were obtained from animal sources such as rooster combs. All the modern products are produced by bacterial fermentation with equine streptococci. ${ }^{14}$ A number of HA filler products are currently available, with FDA indications for one or more of the following: the correction of facial wrinkles and/or folds such as the nasolabial folds, lip augmentation, and volumization of the midface. HA fillers are by far the most popular type of dermal fillers for cosmetic use primarily due to the ability to dissolve the product, usually within 24 hours, with commercially available hyaluronidase.

Another product used for hand augmentation is a powdered form of poly-1-lactic acid (PLLA) that is soluble in sterile water (Sculptra ${ }^{\circledR}$; Galderma Laboratories, Fort Worth, TX, USA). PLLA has been in use since 1960 s for a variety of indications, including resorbable sutures, orthopedic plates, urologic stents, and as a vehicle for vaccines. ${ }^{15,16}$

Over the years, PLLA has demonstrated itself to be a safe, biocompatible, and nonimmunostimulating substance that degrades in the body within a reliable period of time. In the 1990s, microscopic examination of the tissue immediately surrounding the stitches composed of PLLA showed a marked increase in collagen deposition. PLLA was determined to be the active ingredient and in 1999, the first injectable formulation for dermal filling was approved in Europe. The American product was given FDA approval in 2004 for the correction of HIV-associated lipoatrophy and later in 2009 for the correction of nasolabial folds. ${ }^{17}$

PLLA is available in powdered form, and each vial is reconstituted with sterile water. Reconstitution protocols vary, but most practitioners have made the suspension more dilute over the years to increase liquidity and avoid nodules. The current trend is to add at least $7 \mathrm{~mL}$ of water, frequently with an additional amount of lidocaine, both with or without epinephrine for facial and hand injections. ${ }^{18}$ Reconstitution should happen at least 12 hours prior to the planned injection so that the microparticles have enough time to disperse into a smooth suspension. Neocollagenesis with PLLA takes time. The initial volumization effect disappears within several days as the water component is absorbed. The PLLA microparticles are then encapsulated, and neocollagenesis occurs over a period of 4-6 weeks. Most patients require two to three injection sessions spaced $\sim 6$ weeks apart. The duration of PLLA effect is variable, but averages 2 years or more.

\section{Clinical efficacy and safety}

Currently, CaHA (Radiesse ${ }^{\circledR}$; Merz Aesthetics) is the only FDA-approved filler for hand augmentation. The pivotal trial leading to approval consisted of a multicenter, randomized controlled study of 114 patients, the majority being Caucasian females with Fitzpatrick skin types I-III and an 
average age of 54 years. ${ }^{5}$ Eighty-five patients were randomly assigned to immediate treatment, with 29 patients assigned to an untreated control group (delayed treatment) for the first 3 months after which they were crossed over and received treatment. All patients were eligible to receive retreatment at 6 months.

CaHA mixed with $2 \%$ lidocaine $\mathrm{HCl}$ was administered to the dorsum of the hands using a 27-gauge needle in multiple aliquots of $0.5 \mathrm{~mL}$ each with a mean of $2.59 \mathrm{~mL}$ per hand (range 1.4-3.6 mL), and then massaged into place. Efficacy was assessed at 3 months by a blinded investigator using the validated Merz Hand Grading Scale (MHGS) through live evaluation and by the patients using the Global Aesthetic Improvement Scale (GAIS) by comparing their hands to baseline photos. The primary efficacy outcome was $\geq 1$-grade improvement on the MHGS between baseline and 3 months for both hands of the treatment group compared with the control group. Results demonstrated that $75 \%$ of $\mathrm{CaHA}$ patients experienced $\geq 1$-grade improvement compared with $3 \%$ of the control group. The mean change from baseline on the MHGS was 1.1 grade in the CaHA group compared to 0.1 for the control group, which was statistically significant. Results from the GAIS indicated that $98 \%$ of treated patients reported improvement in the appearance of their hands.

Retreatment volume administered averaged $1.63 \mathrm{~mL}$ per hand with a range of 0.7-3.0 mL. Responder rates demonstrated lasting results, with $68 \%$ of patients maintaining $\geq 1$-grade improvement at 12 months postinitial treatment. AEs reported by patients over 12 months posttreatment included swelling (99\%), pain (92\%), redness (81\%), bruising $(73 \%)$, difficulty in performing activities (48\%), itching (46\%), loss of sensation (15\%), and nodules, lumps/bumps $(6 \%)$. AEs considered by patients were primarily mild (12\%) to moderate $(69 \%)$ in severity, and all AEs resolved without sequelae or treatment. The majority of AEs lasted less than 2 weeks; those with duration greater than 2 weeks included swelling (29\%), pain (25\%), nodules, lumps/bumps (7\%), difficulty in performing activities $(6 \%)$, redness $(6 \%)$, and bruising (3\%). The results of this pivotal study demonstrated that CaHA was safe and effective for augmentation of the hands for up to 1 year.

The use of other types of fillers for hand volumization has also been described in the literature. Sadick et al reported on their experience using PLLA for hand rejuvenation. ${ }^{19}$ The results indicated that patients were very satisfied with the treatment, and AEs were mild and transient. Man et al compared small-particle HA (Restylane ${ }^{\circledR}$; Galderma S. A., Lausanne, Switzerland) to collagen (Cosmoplast ${ }^{\circledR}$; Allergan
Inc., Irvine, CA, USA) and found that HA demonstrated increased efficacy compared with collagen, with patient satisfaction similar between groups. ${ }^{20}$ Williams et al found that hands treated with HA generated significant improvements in skin elasticity and surface roughness, with increased hydration, compared to baseline. ${ }^{21}$

\section{Filler choice for hand augmentation}

The injectable agents that are currently being used for hand rejuvenation are all excellent products. HA, CaHA, and PLLA are all biocompatible, simple to administer, safe, and easily tolerated with or without topical numbing cream. When injected with care by an experienced provider, these agents produce a smooth and natural appearance to the dorsal hand with a minimum of bruising, swelling, and discomfort. If overinjection is avoided, these procedures should never cause limitations in hand motion.

All of the injectables stimulate collagen growth to a variable degree. Histological studies demonstrate that PLLA produces the most exuberant response, ${ }^{22}$ but $\mathrm{CaHA}$ and $\mathrm{HA}$ will also cause some proliferation in collagen production. ${ }^{6,23}$ All of these injectables are moldable immediately after injection, and firm massage will usually distribute the agent exactly into the desired area. Even though the hand is a mobile structure, migration of filler has not been described in the literature. AEs that are frequently seen postinjection include edema, erythema, ecchymosis, and pruritus. These are all transient, but the frequency and intensity of AEs vary with the choice of injectable. Although the occurrence is fairly rare, PLLA has been known to cause nodules when used in the hands, which is why its use for hand augmentation lags behind that of CaHA or HA fillers. CaHA causes more swelling and bruising than HA fillers, but it replaces volume efficiently, so it is still frequently used in the hands.

Choice of filler and injection technique should always be determined based on in-depth consultation, examination of the individual patient, and, occasionally, on patient preferences. Many patients prefer a filler that is reversible, in which case the choice is limited to the available HA products. Typically, these are the patients who are having the procedure done for the first time, and reversibility gives them peace of mind. Any of the commercially available HA fillers will work well to replace volume in the hand, but the best choice will be determined by severity of volume loss as measured by the MHGS (Figure 1). ${ }^{24}$

The protocol that the author prefers after treating over 100 patients for hand rejuvenation is to use diluted VYC-20L HA gel (Juvéderm Voluma ${ }^{\circledR}$ XC; Allergan Inc.) 
Merz Hand Grading Scale

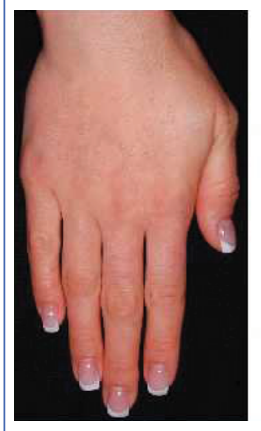

No loss of fatty tissue

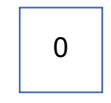

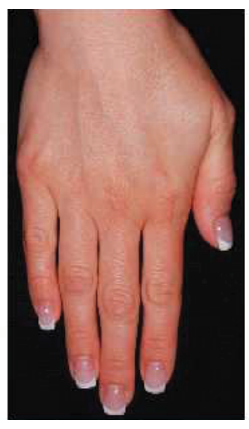

Mild loss of fatty tissue; slight visibility of veins

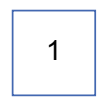

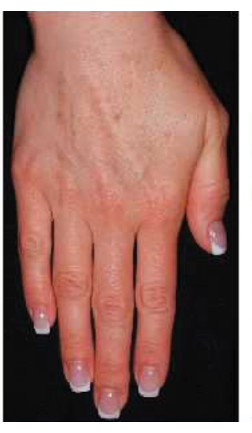

Moderate loss of fatty tissue; mild visibility of veins and tendons

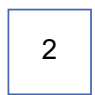

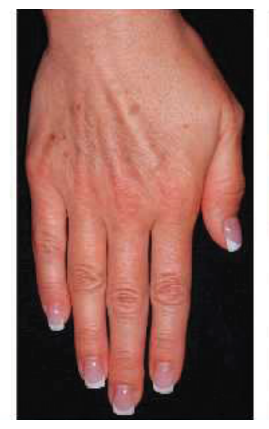

Severe loss of fatty tissue; moderate visibility of veins and tendons

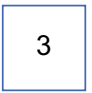

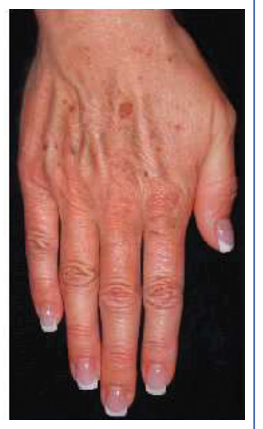

Very severe loss of fatty tissue; marked visibility of veins and tendons

4

Figure I Merz Hand Grading Scale

Note: Copyright 2007 Merz Pharmaceuticals, reproduced with permission.

off-label when treating MHGS grades 2 and 3. The author prefers to dilute it $1: 1$ with $0.8 \mathrm{~mL}$ saline and $0.2 \mathrm{~mL}$ of $1 \%$ lidocaine with 1:100,000 parts epinephrine. Since VYC-20L HA gel already contains some lidocaine, the diluent is added primarily for the mild vasoconstrictive effect of the lowconcentration epinephrine. Getting a smooth, dilute mixture involves connecting the filler syringe to the diluent syringe with a Luer Lock connector and mixing the products back and forth at least 20 times. Dilution improves distribution of the injected product and helps to ensure that the final contour is as smooth and natural in appearance as possible. In my personal experience, diluted VYC-20L HA gel in the hands lasts up to 2 years. The other advantage to dilution is that smaller gauge needles can be used, decreasing the risk of bruising and embolic AEs. The author typically uses a 30-gauge, $0.5-1$ inch needle to administer the filler. This is a moot point when using cannulas to deliver the product, since undiluted VYC-20L HA gel will easily go through a 27 -gauge cannula. When treating more severe volume loss such as MHGS 4, undiluted VYC-20L HA gel provides the necessary lift for an aesthetically satisfactory result, but the injector has to take care to ensure that the postinjection dorsum is not irregular in contour. VYC-20L HA gel should be injected slowly and carefully, avoiding large boluses and overinjection. Postinjection massage is recommended to smooth out the product. Hands that are MHGS 4 often require more than one syringe per hand.

Some injectors prefer to use CaHA for hand augmentation rather than HA fillers due to the comfort of using an FDA-approved product for the indication and consider the opaque color of the filler an advantage in avoiding the Tyndall effect. Another advantage to CaHA is its high G' (or stiffness), giving it the best lifting ability of any filler. This makes CaHA economically advantageous because less amount of the product is needed to produce significant lift for patients with advanced volume loss. When injecting CaHA, most practitioners will dilute the product 1:1 with lidocaine or a mixture of lidocaine and saline. The author's personal preference is to use the same diluent with $\mathrm{CaHA}$ as he does with HA - saline and lidocaine with epinephrine. The dilution technique for CaHA is the same as described for VYC-20L HA gel.

The author's hesitation with using CaHA for hand augmentation is the duration of effect. The pivotal study for FDA approval demonstrated that $68 \%$ of patients had a 1-grade or better improvement at 12 months, which mirrors the author's experience with CaHA for facial rejuvenation. The author's personal experience of injecting the product daily for over a decade is that there is significant variability in the duration of effect. The result lasts 6 months in some patients, and others get twice that. Long-term studies comparing duration of CaHA and VYC-20L HA gel in the hands have yet to be performed, but, extrapolating from the VYC-20L HA gel data for the face, it is reasonable to conclude that it will outlast CaHA. That being said, the author still sometimes uses CaHA because it is cost effective, but patients need to understand that they may need to return for a repeat treatment within 8-12 months. 


\section{Anatomy}

A thorough understanding of anatomy is necessary for successful rejuvenation of the hands with fillers. Bidic et al conducted a histological and ultrasound analysis of the dorsum of the hand and found three distinct fatty-areolar laminae that they called the dorsal superficial, the dorsal intermediate, and the dorsal deep lamina. ${ }^{25}$ The intermediate and deep laminar layers are separated from the lamina superficial to it by distinct fascial layers, called the dorsal superficial fascia and the dorsal intermediate fascia, respectively. The third fascial layer, the dorsal deep fascia, separates the floor of the dorsal deep lamina from the dorsal interosseous muscles and the metacarpal bones. The dorsal intermediate fascia is an extension of the antebrachial fascia of the forearm, and the dorsal deep fascia is contiguous with the periosteum overlying the metacarpals. The sensory nerves and dorsal veins are contained within the dorsal intermediate lamina and the extensor tendons reside in the dorsal deep lamina. No structures are apparent in the dorsal superficial lamina. Adhesions between fascial layers were determined to be fascial sheaths surrounding arteries and veins running through fascial septa. These adhesions are located within the dorsal superficial lamina, numbered 8-12 per hand, and inserted into the dermis, presumably to supply the subdermal plexus.

It was noted that a greater amount of superficial fat in the dorsal superficial lamina was apparent in hands from cadavers with a body mass index (BMI) $>30 \mathrm{~kg} / \mathrm{m}^{2}$, which masked the underlying structures such as veins and tendons, compared to hands from cadavers $<20 \mathrm{BMI} \mathrm{kg} / \mathrm{m}^{2}$. In the hands from the lower BMI cadavers, the dermis and dorsal superficial fascia appeared relatively contiguous.

\section{Injection technique based on the author's personal experience}

There are several techniques for injecting the dorsal hand with fillers regardless of filler type used. Some injectors prefer to inject a small amount of lidocaine at the entry points for pain control. Topical topical triple anesthetic cream also helps with discomfort. The author tries to use lidocaine injection only with patients who are particularly sensitive. Anesthetic injection often obscures the contours being corrected, so the author avoids it whenever possible. The author also tries to avoid topical anesthetic cream because the author finds that it causes mild edema, increasing the possibility of undercorrection. The hand should be comfortably positioned on a draped surface. The author prefers the patient to be sitting up with the hands beneath the heart so that veins are clearly visible. Prior to injection, the skin should be cleansed with alcohol or a chlorhexidine solution. During injection, the cleansing agent should be reapplied frequently. The author often sees novice injectors contaminate the skin and neglect to reapply alcohol, which may increase the risk of complications.

Some injectors prefer to use blunt cannulas when augmenting the hand whereas others prefer to use needles. ${ }^{1}$ When using a cannula, some practitioners prefer to have their entry point just distal to the wrist crease, medially at the carpal-metacarpal junction. The product is administered in a fanning technique from that one entry point. Other injectors prefer to make four or five entry points in the web space of each metacarpal-phalange junction and inject the product in a linear threading technique or small boluses. ${ }^{1}$

There are two primary techniques for hand augmentation using needles. The first, popularized by Busso in 2007, is to tent the skin, place a large bolus of product over the tendons around the midpoint of the dorsal hand, and massage firmly until the product distributes evenly and the hand regains a natural contour. ${ }^{26}$ The second and currently more popular technique is to inject into the web space between the phalanges (Figure 2). The author prefers needles for the precision of effect and use this second technique whether the author is injecting CaHA or HA. The author believes injecting between the phalanges results in more significant correction for the volume used with minimal swelling because there is less massage necessary.

Whether using needles or cannulas, most practitioners aim for the dorsal superficial lamina as it is a relatively sparsely vascularized space above the dorsal intermediate lamina that contains the major veins of the hand. At the entry points, the skin is tented up to open the dorsal superficial lamina space so that the needle or cannula reliably enters that plane. The

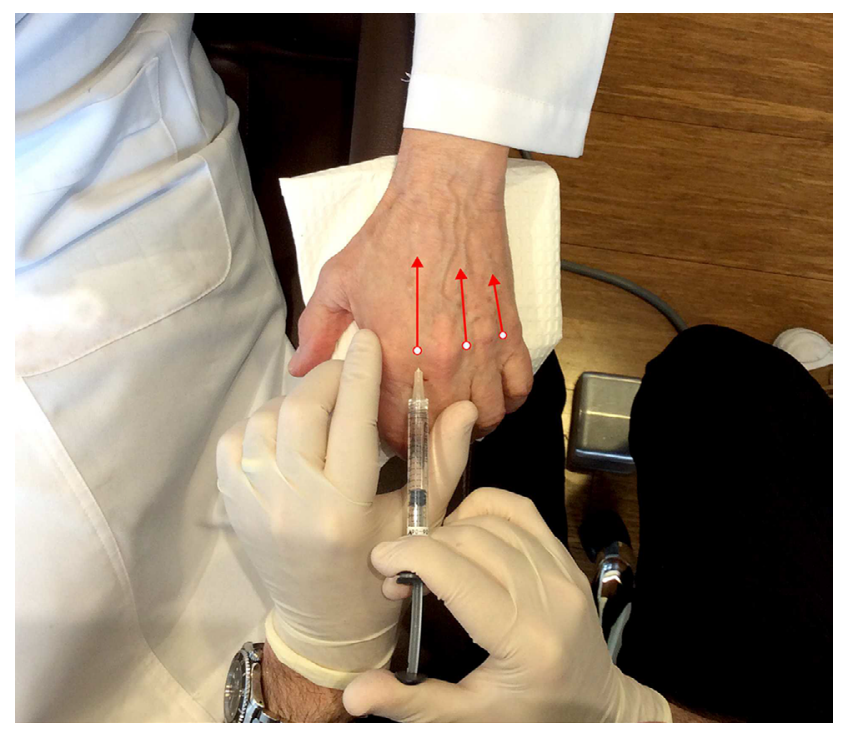

Figure 2 Injection technique: after tenting the skin, the needle is inserted into the space between each metacarpal joint and advanced along the path of the red arrows. Product is injected as the needle is withdrawn. 
intrametacarpal spaces can be thought of as elongated triangles with the most narrow point proximally located. The author fills each space in one to two passes, taking care not to overfill the narrow, proximal point. The aesthetic goal of this procedure is to minimize the contour transitions around each vein or tendon. A natural result may have visible tendons and veins, but the spaces next to those structures are filled in and transitions are smooth (Figure 3). The aim of hand augmentation is to try to camouflage the veins and tendons. In a successful procedure, shadowing is minimized and those structures are less evident to the eye of the observer. The number of passes should be minimized to decrease the risk of bruising. Most patients will get good correction with 1-3 mL of HA or CaHA per hand. After injection, the hands are massaged to evenly distribute the filler throughout the dorsum. The author usually asks the patient to make a fist thereby tensing the skin, and massages the dorsum of the hand with his thumbs (Figure 4). Arnica cream or ultrasound gel allow for easier massage.

After injection, the patient should ice her hands for the rest of the day. She should also elevate her hands as much as possible (day and night) and try to avoid salty food for the next 2-3 days. The author recommends avoiding alcohol, strenuous exercise, and ibuprofen or aspirin for a day posttreatment. She should also avoid intense work with her hands for a week or so.

ADs resulting from injecting filler into the hands are similar to those found when using filler for facial injections.
Practitioners are most concerned with embolic phenomena causing ischemia and necrosis of the skin or worse. To minimize the risk, the author advocates the adoption of common sense universal injection precautions. These are the same precautions that are used to optimize safety with facial filler injections (Table 1). Other AEs to watch for in the hands are: bruising, swelling, erythema, pruritus, and functional compromise. These are all transient effects. Swelling is the most frequently encountered and is found to some degree in most patients. The degree of swelling is related to the amount of filler injected (although CaHA seems to cause more swelling than HA at equivalent volume), rewarding the conservative injector. It is preferable to bring patients back for additional treatment than to inject too much in one session. Most swelling occurs immediately, persists for a few days, and then resolves. Occasionally, some patients experience delayed swelling several days to a week after injection. For swelling that persists for longer than 2 weeks, a short, tapered dose of oral methylprednisolone will usually resolve the issue.

Bruising after hand rejuvenation by filler injection is common, but is usually mild and short lived. More significant bruising can be treated with a pulsed dye laser such as the V Beam (Syneron Candela Corp, Irvine, CA, USA) to decrease the intensity and duration of the ecchymosis. Persistent erythema can be treated this way as well.

The author has not yet seen serious or long-term complications from hand rejuvenation with fillers, although granuloma

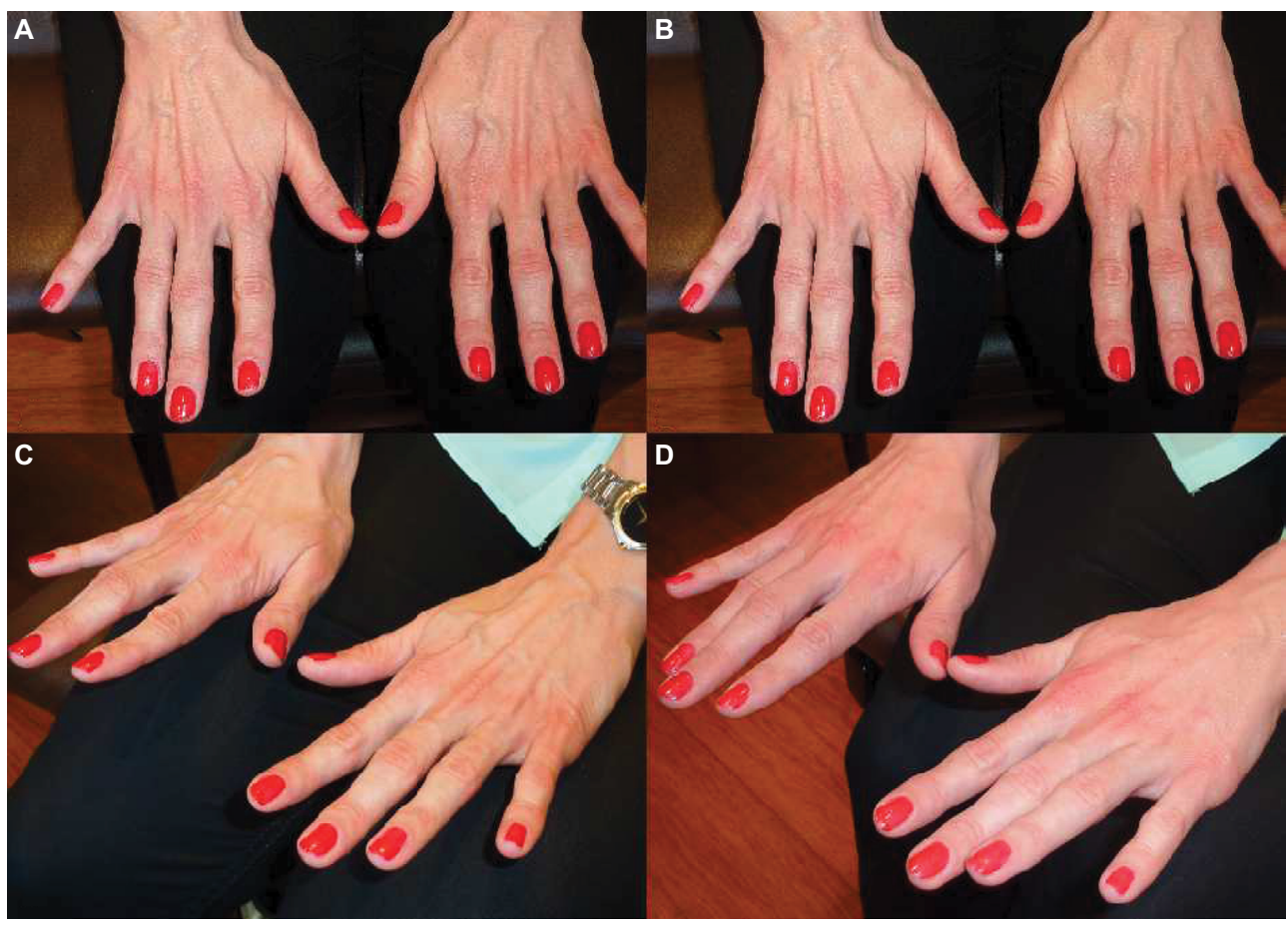

Figure 3 Before (A and $\mathbf{C}$ ) and one year after (B and $\mathbf{D})$ hand augmentation with $4 \mathrm{cc}$ of diluted VYC-20L HA gel. In my experience, the result lasts for an average of one and a half years. 


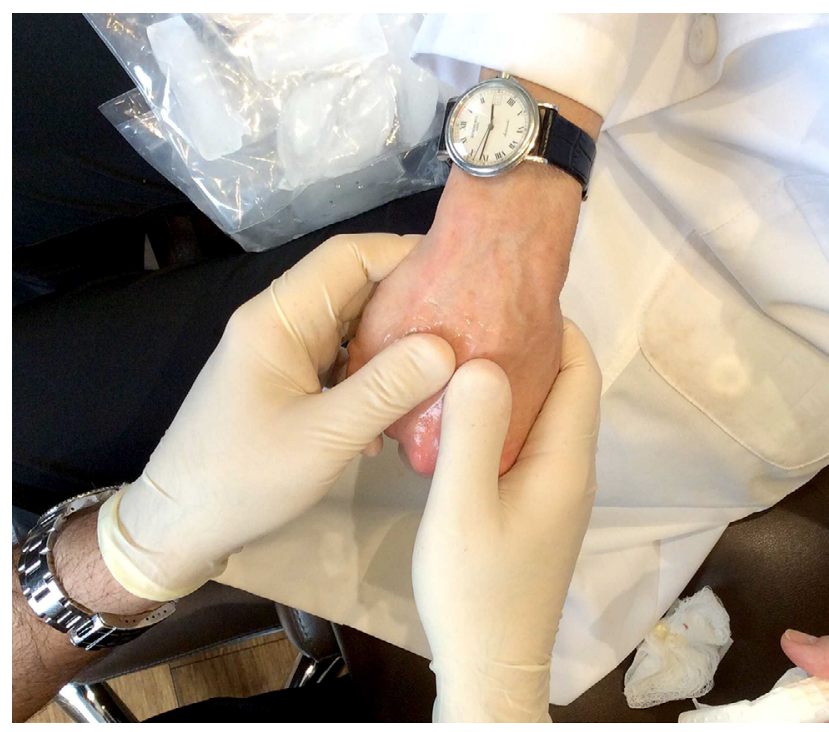

Figure 4 Vigorous massage after injection blends the injected product into the empty spaces of the hand so that the final contours are smooth and natural in appearance.

Table I Injection tips to decrease the potential for adverse events during hand rejuvenation treatment using fillers

I. Needles should be as small as possible so that the flow rate of the filler is low.

2. Fillers should always be injected slowly, gently, and under low pressure. Gentle injection technique may prevent many complications.

3. The needle should be advanced through the skin slowly and filler should be injected in a retrograde fashion, while the needle is moving out of the skin. This technique safeguards that even if the tip of the needle is inside the lumen of a vessel at some point, only a tiny amount of filler will enter the vessel as the needle will be out in the next moment.

4. Small volumes of filler should be introduced with each injection.

5. Use ice prior to injection. Ice decreases pain and shrinks blood vessels making them less likely to be punctured.

formation has been reported in the literature. ${ }^{27}$ The management of a granuloma in the hands should, initially, be the same as that of a granuloma in the face. HA granulomas should be treated with hyaluronidase and steroids (the author prefers a mixture containing equal parts of Kenalog 40, 5-fluorouracil, and dexamethasone), whereas non-HA filler granulomas can be treated with steroid injection only. Oral steroids and antibiotics may be used for recurrent granulomas, and surgical excision should be performed only if all other options fail. ${ }^{27}$

\section{Patient satisfaction}

Patient satisfaction is high for hand rejuvenation treatment across all of the fillers described in the literature. . $^{19,21,28,29}$ Marmur et al conducted a small pilot study investigating patient satisfaction of CaHA for hand augmentation. ${ }^{29}$ All five patients in the study were very satisfied at 24 weeks posttreatment. Marmur observed that swelling that lasted $\geq 1$ week resulted in decreased patient satisfaction and suggested treating the swelling with methylprednisolone, which restored patient satisfaction. In a larger study of 101 patients, Busso found $78 \%$ and $76 \%$ of patients treated with $\mathrm{CaHA}$ for hand rejuvenation were satisfied or extremely satisfied at 3 and 6 months, respectively, and $66 \%$ stated they were likely or extremely likely to return for future treatment. ${ }^{28}$

\section{Conclusion}

When properly performed by a skilled practitioner, rejuvenation of the hands with dermal fillers is a simple procedure with high patient satisfaction and a low rate of complications. Although the majority of patients requesting cosmetic procedures look to rejuvenate the face, aging hands are just as visible and clear indicators of age. Hand rejuvenation enables the cosmetic injector to more fully address their patient's aesthetic concerns.

\section{Acknowledgment}

The author wishes to thank Diana Romero, PhD, for editorial assistance.

\section{Disclosure}

Dr Rivkin is a speaker and trainer for Allergan, Inc., Merz Aesthetics, Suneva Medical Inc. (San Diego, CA, USA), and Galderma S. A. The author reports no other conflicts of interest in this work.

\section{References}

1. Lefebvre-Vilardebo M, Trevidic P, Moradi A, Busso M, Sutton AB, Bucay VW. Hand: clinical anatomy and regional approaches with injectable fillers. Plast Reconstr Surg. 2015;136(Suppl 5):258S-275S.

2. Shamban AT. Combination hand rejuvenation procedures. Aesthet Surg J. 2009;29(5):409-413.

3. Fournier PF. Who should do syringe liposculpture? J Dermatol Surg Oncol. 1988;14(10):1055-1056.

4. Coleman SR. Hand rejuvenation with structural fat grafting. Plast Reconstr Surg. 2001:110(7):1731-1744.

5. Radiesse ${ }^{\mathbb{1}}$ injectable implant [instructions for use for the dorsum of the hand] Franksville, WI: Merz Aesthetics; 2016.

6. Berlin AL, Hussain M, Goldberg DJ. Calcium hydroxylapatite filler for facial rejuvenation: a histologic and immunohistochemical analysis. Dermatol Surg. 2008;34(Suppl 1):S64-S67.

7. Marmur ES, Phelps R, Goldberg DJ. Clinical, histologic and electron microscopic findings after injection of a calcium hydroxylapatite filler. J Cosmet Laser Ther. 2004;6(4):223-226.

8. Jansen DA, Graivier MH. Evaluation of a calcium hydroxylapatite-based implant (Radiesse) for facial soft-tissue augmentation. Plast Reconstr Surg. 2006;118(Suppl 3):22S-30S.

9. Emer J, Sundaram H. Aesthetic applications of calcium hydroxylapatite volumizing filler: an evidence-based review and discussion of current concepts: Part 1 of 2. J Drugs Dermatol. 2013;12(12):1345-1354.

10. Jacovella PF. Calcium hydroxylapatite facial filler (Radiesse): indications, technique, and results. Clin Plast Surg. 2006;33(4):511-523.

11. Bass LS, Smith S, Busso M, McClaren M. Calcium hydroxylapatite (Radiesse) for treatment of nasolabial folds: long-term safety and efficacy results. Aesthet Surg J. 2010;30(2):235-238.

12. Tezel A, Frederickson GH. The science of hyaluronic acid dermal fillers. $J$ Cosmet Laser Ther. 2008;10(1):35-42. 
13. Laurent TC, editor. The Chemistry, Biology and Medical Applications of Hyaluronan and its Derivatives. Wenner-Gren International Series, Vol 72. London: Portland Press; 1998.

14. Andre P. Hyaluronic acid and its use as a "rejuvenation" agent in cosmetic dermatology. Semin Cutan Med Surg. 2004;23(4):218-222.

15. Humble G, Mest D. Soft tissue augmentation using Sculptra. Facial Plast Surg. 2004;20(2):157-163.

16. Suuronen R, Haers PE, Lindqvist C, Sailer HF. Update on bioresorbable plates in maxillofacial surgery. Facial Plast Surg. 1999;15(1): 61-72.

17. Keni SP. SidleDM. Sculptra(injectablepoly-L-lacticacid). FacialPlast Surg Clin North Am. 2007;15(1):91-97.

18. Lorenc ZP, Greene T, Gottschalk RW. Injectable poly-1-lactic acid: understanding its use in the current era. J Drugs Dermatol. 2016;15(6): 759-762.

19. Sadick NS, Anderson D, Werschler WP. Addressing volume loss in hand rejuvenation: a report of clinical experience. J Cosmet Laser Ther. 2008; 10(4):237-241.

20. Man J, Rao J, Goldman M. A double-blind, comparative study of nonanimal-stabilized hyaluronic acid versus human collagen for tissue augmentation of the dorsal hands. Dermatol Surg. 2008;34(8):1026-1031.

21. Williams S, Tamburic S, Stensvik H, Weber M. Changes in skin physiology and clinical appearance after microdroplet placement of hyaluronic acid in aging hands. J Cos Dermatol. 2009;8(3):216-225.
22. Stein P, Vitavska O, Kind P, Hoppe W, Wieczorek H, Schürer NY. The biological basis for poly-L-lactic acid-induced augmentation. J Dermatol Sci. 2015;78(1):26-33.

23. Wang F, Garza LA, Kang S, et al. In vivo stimulation of de novo collagen production caused by cross-linked hyaluronic acid dermal filler injections in photodamaged human skin. Arch Dermatol. 2007;143(2): 155-163.

24. Cohen JL, Carruthers A, Jones DH, et al. A randomized, blinded study to validate the Merz Hand Grading Scale for use in live assessments. Dermatol Surg. 2015;41(Suppl 1):S384-S388.

25. Bidic SM, Hatef DA, Rohrich RJ. Dorsal hand anatomy relevant to volumetric rejuvenation. Plast Reconstr Surg. 2010;126(1):163-168.

26. Busso M, Applebaum D. Hand augmentation with Radiesse (calcium hydroxylapatite). Dermatol Ther. 2007;20(6):385-387.

27. Lee JM, Kim YJ. Foreign body granulomas after the use of dermal fillers: pathophysiology, clinical appearance, histologic features, and treatment. Arch Plast Surg. 2015;42(2):232-239.

28. Busso M, Moers-Carpi M, Storck, R, Ogilvie P, Ogilvie A. Multicenter, randomized trial assessing the effectiveness and safety of calcium hydroxylapatite for hand rejuvenation. Dermatol Surg. 2010;36(Suppl 1): 790-797.

29. Marmur ES, Al Quran H, de Sa Earp AP, Yoo SY. A five-patient satisfaction pilot study of calcium hydroxylapatite injection for treatment of aging hands. Dermatol Surg. 2009;35(12):1978-1984.
Clinical, Cosmetic and Investigational Dermatology

\section{Publish your work in this journal}

Clinical, Cosmetic and Investigational Dermatology is an international, peer-reviewed, open access, online journal that focuses on the latest clinical and experimental research in all aspects of skin disease and cosmetic interventions. This journal is included on PubMed. The manuscript management system is completely online

\section{Dovepress}

and includes a very quick and fair peer-review system, which is all easy to use. Visit http://www.dovepress.com/testimonials.php to read rea quotes from published authors 